\title{
Endoscopic transforaminal lumbar interbody fusion without general anesthesia: operative and clinical outcomes in 100 consecutive patients with a minimum 1-year follow-up
}

\author{
John Paul G. Kolcun, BS, ${ }^{1}$ G. Damian Brusko, BS,1 Gregory W. Basil, MD, ${ }^{1}$ Richard Epstein, MD, ${ }^{2}$ \\ and Michael Y. Wang, MD'
}

Departments of ${ }^{1}$ Neurological Surgery and ${ }^{2}$ Anesthesiology, University of Miami Miller School of Medicine, Miami, Florida

OBJECTIVE Open spinal fusion surgery is often associated with significant blood loss, postoperative pain, and prolonged recovery times. Seeking to minimize surgical and perioperative morbidity, the authors adopted an endoscopic minimally invasive transforaminal lumbar interbody fusion (MIS-TLIF) technique performed without general anesthesia. In this report, they present data on the first 100 patients treated with this procedure.

METHODS The authors conducted a retrospective review of the first 100 patients who underwent awake endoscopic MIS-TLIF at a single institution between 2014 and 2017. Surgery was performed while the patient was sedated but without intubation or the use of general anesthetic or narcotic agents. Long-lasting (liposomal) bupivacaine was used for local analgesia. The discectomy and placement of an expandable interbody graft were performed endoscopically, followed by percutaneous pedicle screw implantation. Inclusion criteria for the procedure consisted of diagnosis of degenerative disc disease with grade I or II spondylolisthesis and evidence of spinal stenosis or nerve impingement with intractable symptomatology.

RESULTS Of the first 100 patients, 56 were female and 44 were male. Single-level fusion was performed in 84 patients and two-level fusion in 16 patients. The most commonly fused level was $L 4-5$, representing $77 \%$ of all fused levels. The mean ( \pm standard deviation) operative time was $84.5 \pm 21.7$ minutes for one-level fusions and $128.1 \pm 48.6$ minutes for two-level procedures. The mean intraoperative blood loss was $65.4 \pm 76.6 \mathrm{ml}$ for one-level fusions and $74.7 \pm 33.6 \mathrm{ml}$ for two-level fusions. The mean length of hospital stay was $1.4 \pm 1.0$ days. Four deaths occurred in the 100 patients; all four of those patients died from complications unrelated to surgery. In $82 \%$ of the surviving patients, 1-year follow-up Oswestry Disability Index (ODI) data were available. The mean preoperative ODI score was $29.6 \pm 15.3$ and the mean postoperative ODI score was $17.2 \pm 16.9$, which represents a significant mean reduction in the ODI score of -12.3 using a two-tailed paired t-test $(p=0.000001)$. In four cases, the surgical plan was revised to include general endotracheal anesthesia intraoperatively and was successfully completed. Other complications included two cases of cage migration, one case of osteomyelitis, and one case of endplate fracture; three of these complications occurred in the first 50 cases.

CONCLUSIONS This series of the first 100 patients to undergo awake endoscopic MIS-TLIF demonstrates outcomes comparable to those reported in our earlier papers. This procedure can provide a safe and efficacious option for lumbar fusion with less morbidity than open surgery. Further refinements in surgical technique and technologies will allow for improved success.

https://thejns.org/doi/abs/10.3171/2018.12.FOCUS18701

KEYWORDS lumbar fusion; minimally invasive transforaminal lumbar interbody fusion; awake fusion; enhanced recovery after surgery; length of stay; outcomes

ABBREVIATIONS ERAS = enhanced recovery after surgery; GETA = general endotracheal anesthesia; LOS = length of stay; MIS = minimally invasive; ODI = Oswestry Disability Index; rhBMP-2 = recombinant human bone morphogenetic protein-2; TLIF = transforaminal lumbar interbody fusion.

ACCOMPANYING EDITORIAL DOI: 10.3171/2019.1.FOCUS1945.

SUBMITTED December 1, 2018. ACCEPTED December 26, 2018.

INCLUDE WHEN CITING DOI: 10.3171/2018.12.FOCUS18701. 
$\mathrm{T}$ HE rate of spinal fusion procedures in developed nations has steadily increased in recent decades. ${ }^{9}$ This increase is a result of aging populations in these countries and a consequent rise in the incidence of degenerative spinal pathology, ${ }^{4,10}$ better evidence for the efficacy of spinal fusion in appropriate patients, ${ }^{7,8}$ and new techniques in minimally invasive (MIS) spine surgery. ${ }^{1}$ These MIS techniques have resulted in less pain, reduced blood loss, and shorter hospital stays for patients, enabling spinal surgeons to offer fusion surgery to those who may otherwise be wary of it.

In the developing medical culture, which emphasizes quality and financial austerity, methods to reduce hospital length of stay (LOS) in particular benefit both patients and hospital administrations. The principles of enhanced recovery after surgery (ERAS) ${ }^{6}$ can and should be applied to spinal fusion, for which patients may fear a prolonged postoperative inpatient recovery period that could be extended by reduced mobility and reliance on narcotic analgesia. ${ }^{11}$ The application of ERAS principles shortens the inpatient recovery period for patients and reduces the acute care costs for hospital systems. ${ }^{12}$

We previously described our early experiences with awake transforaminal lumbar interbody fusion (TLIF) - a procedure that involves endoscopic discectomy visualization, expandable graft technology, osteobiologic agents, percutaneous instrumentation, and long-acting local anesthesia-performed on consciously sedated patients and not requiring general endotracheal anesthesia (GETA). ${ }^{13,14}$ Since that time, we have continued to improve and expand this technique to incorporate the lessons learned throughout a larger series of cases.

While we have developed and implemented an ERAS protocol for lumbar spinal fusion at our institution, awake TLIF represents a specific procedure distinct from that protocol. In this report, we detail the technical refinements of the procedure and the clinical results in the first 100 consecutively treated patients, who underwent surgery before implementation of our institution's ERAS program.

\section{Methods \\ Patient Series}

We conducted a retrospective review of the first 100 patients who underwent single- or multilevel awake endoscopic MIS-TLIF at the University of Miami Hospital within a 3-year period (July 2014-August 2017). Baseline demographic characteristics were recorded, including age, sex, and the level of fusion that was performed. Subsequent intraoperative data, such as blood loss and operative time, were obtained from the patients' medical records. Oswestry Disability Index (ODI) data were recorded at the patient's first preoperative visit and at 3-month intervals thereafter. The preoperative ODI was compared to the most recent ODI available 1 year postoperatively or later.

\section{Anesthetic Technique}

Although we have previously described the anesthetic and surgical technique for this procedure, ${ }^{2,14}$ we review it again here. Surgery was performed while the patient was sedated, but without intubation or the use of general anes- thetic or narcotic agents. Due to prone positioning of the patient and lack of an advanced airway, supplemental oxygen via a nasal cannula or a face mask and careful anesthesia monitoring are critical. Sedation was accomplished using a combination of propofol (Diprivan, Fresenius Kabi USA) and ketamine. Continuous communication between the surgeon and the anesthesiologist is critical to achieve optimal sedation.

Ideally, patients are placed under light to moderate sedation, allowing the surgeon to benefit from patient feedback, which may indicate the proximity of instruments to critical neural elements. Long-lasting (liposomal) bupivacaine (Exparel, Pacira Pharmaceuticals) was used for local analgesia; this assisted in both intraoperative and postoperative pain management.

Based on four cases in which the patients required conversion to GETA, as discussed in the Results section, adjustments were made to our preoperative protocol. Specifically, all subsequent patients were given oxymetozoline spray preoperatively to avoid epistaxis. Additionally, ondansetron along with glycopyrrolate was added to the preoperative medication regimen to avoid intraoperative emesis.

\section{Surgical Technique}

The patients were placed prone on a Jackson table with their arms in the "Superman" position. Localization was accomplished using a spinal needle guided by both anteroposterior and lateral fluoroscopy to access the disc space via Kambin's triangle at the level of interest. A Nitinol wire was placed into the disc space and successive dilation was performed to accommodate the 8-mm working channel (Joimax, Inc.). An endoscope was then inserted and key anatomical landmarks, including the exiting and traversing nerve roots, were visualized.

Bone and cartilaginous decompression was then accomplished under direct visualization by using a combination of bipolar cautery, pituitary rongeurs, osteotomes, curettes, and powered drills. Adequate decompression was confirmed using the endoscope to visualize the neural elements. Discectomy and endplate preparation were then performed using a combination of bipolar cautery, pituitary rongeurs, curettes, and stainless steel brushes attached to a powered drill. Following two early cases of cage migration, additional measures were taken to confirm sufficient endplate preparation. Specifically, an inflatable balloon was placed into the disc space and subsequently expanded using radiopaque contrast material. Once inflated, an anteroposterior radiograph was obtained and, if there appeared to be residual cartilaginous endplate, additional endplate preparation was performed.

Once the disc space had been prepared, $2.1 \mathrm{mg}$ of recombinant human bone morphogenetic protein-2 (rhBMP-2; Infuse, Medtronic Sofamor Danek) was placed into the anterior disc space. Adequate anterior positioning was verified by pretreating the rhBMP-2 with a small amount of iohexol (Omnipaque, GE Healthcare), allowing visualization with fluoroscopy. Next, a 22-mm or 25$\mathrm{mm}$ OptiMesh (Spineology) cage was placed into the disc space and progressively filled with premachined allograft matrix. Satisfactory restoration of disc height and reduc- 

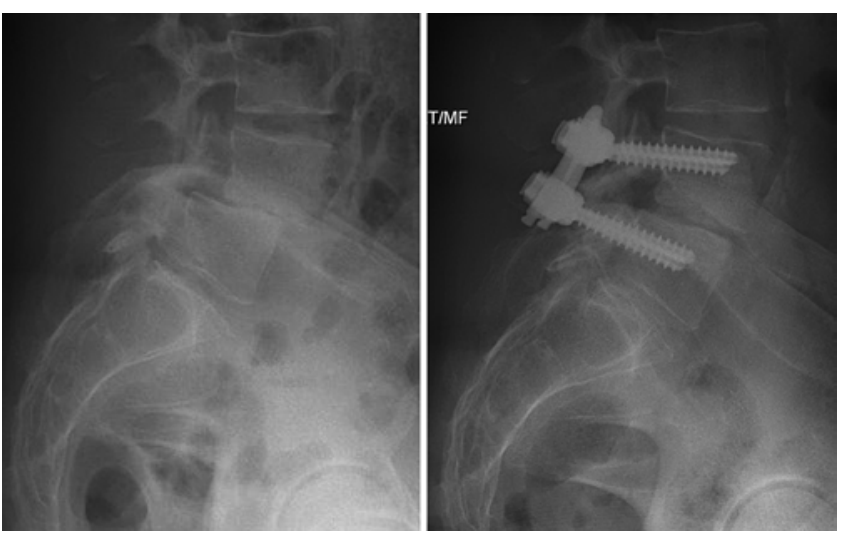

FIG. 1. Representative case of a patient before and after awake TLIF. Preoperative (left) and postoperative (right) radiographs demonstrating proper fusion and reduction of spondylolisthesis.

tion in spondylolisthesis was verified as the graft was progressively filled (Fig. 1).

Percutaneous pedicle screws were then placed using Jamshidi needles under anteroposterior and lateral fluoroscopic guidance. Each track was injected with approximately $5 \mathrm{ml}$ of liposomal bupivacaine (Exparel, diluted 1:2 to a total volume of $10 \mathrm{ml}$ ) under pressure into the posterior musculature. Guidewires were inserted through the cannula of the Jamshidi needles, allowing placement of an awl and subsequently a tap. Screws, 6 or $7 \mathrm{~mm}$ in diameter, were then selected and inserted into the pedicles. Following fluoroscopic verification of screw position, connecting rods were placed bilaterally and subfascially. The set screws were then placed and given a final tightening. Final imaging was performed, and the incisions were closed using a 3-0 Monocryl suture in a figure-eight fashion. Note that the inclusion of Infuse, OptiMesh, and Exparel as applied in this procedure is considered off-label use.

\section{Data Analysis}

Statistical analysis of all data was conducted using Microsoft Excel data analysis tools and a two-sample paired t-test. A p value $<0.05$ was considered statistically significant.

\section{Results}

Clinical and demographic characteristics in the first 100 patients to undergo awake TLIF can be found in Table 1 . A total of 56 patients were female and 44 were male. The patients' average age was $66.0 \pm 11.0$ years (range 28-88 years). Single-level fusion was performed in 84 patients and two-level fusion in 16 patients. The most commonly fused level was L4-5, representing 77\% of all fused levels.

The mean operative time was $84.5 \pm 21.7$ minutes for one-level fusions and $128.1 \pm 48.6$ minutes for two-level fusions. The mean intraoperative blood loss was $65.4 \pm$ $76.6 \mathrm{ml}$ for one-level fusions and $74.7 \pm 33.6 \mathrm{ml}$ for twolevel fusions. The mean LOS was $1.4 \pm 1.0$ days.

There was a total of four deaths in these 100 patients. These patients died from medical causes unrelated to surgery or spinal pathology, at some time after hospital discharge and short-term follow-up in the clinic. The deaths
TABLE 1. Demographic and clinical characteristics in 100 patients who underwent awake endoscopic MIS-TLIF

\begin{tabular}{cc}
\hline Characteristic & Value \pm SD \\
\hline Age (yrs) & \\
\hline Mean & $66.0 \pm 11.0$ \\
\hline Range & $28-88$ \\
\hline Sex (no. of patients) & 44 \\
\hline Male & 56 \\
\hline Female & 116 \\
\hline No. of levels fused & \\
\hline Mean op time (mins) & $84.5 \pm 21.7$ \\
\hline 1-level & $128.1 \pm 48.6$ \\
\hline 2-level & $65.4 \pm 76.6$ \\
\hline Mean intraop EBL (ml) & $74.7 \pm 33.6$ \\
\hline 1-level & $1.4 \pm 1.0$ \\
\hline 2-level & \\
\hline Mean hospital LOS (days) & $29.6 \pm 15.3$ \\
\hline Mean ODl score & $17.2 \pm 16.9$ \\
\hline Preop & $-12.3^{*}$ \\
\hline Postop &
\end{tabular}

$\mathrm{EBL}=$ estimated blood loss.

* Significant at $p=0.000001$.

were discovered by us incidentally during attempted contacts for clinical follow-up, and unfortunately prevented long-term follow-up in these four patients. Eighty-two percent of the surviving patients had follow-up ODI data at a minimum of 1 year postoperatively. The mean preoperative ODI score was $29.6 \pm 15.3$, whereas the mean postoperative ODI score was $17.2 \pm 16.9$. This difference amounts to a statistically significant mean reduction in ODI of -12.3 using a two-tailed paired t-test $(\mathrm{p}=0.000001)$.

In four cases in this series, sedation was converted to GETA intraoperatively. The reasons for conversion were as follows: two patients had emesis, one had epistaxis, and one experienced extreme anxiety. One of the patients with emesis had a known medical history of active reflux. All four of these procedures were successfully completed on the same day using GETA. Adjustments to the protocol were made based on these complications and applied to subsequent cases.

Other complications included two cases of cage migration, one case of osteomyelitis, and one case of endplate fracture. Of these four acute surgical complications, three occurred in the first 50 cases. There were no cases of delayed nonunion or hardware failure over an average radiographic follow-up of 14.6 months among the surviving patients. This was assessed by postoperative flexionextension imaging, which clearly demonstrated radiopaque allograft through the interbody space, in conjunction with the absence of clinical signs of nonunion (for example, worsening axial back pain).

\section{Discussion}

This extended series demonstrates operative and clini- 
cal outcomes comparable to our previously reported findings. ${ }^{13}$ Since our implementation of this technique, the pursuit of rapid recovery for major spine surgery has advanced considerably. This progress has been driven by an increased emphasis on quality enhancement and cost reduction to determine reimbursement as well as greater patient expectations for shorter and more comfortable recovery periods. This trend is further reflected by a growing number of ERAS protocols in a variety of surgical subspecialties. $^{5}$

\section{Operative and Clinical Outcomes}

The reductions in mean operative time, blood loss, and hospital LOS in this extended series, relative to conventional MIS-TLIF, as seen in the literature, are consistent with the reductions we reported in our previous smaller study. 3,5 These low rates were observed in the general sample as well as in one- and two-level cases specifically.

At a minimum 1-year follow-up, there have been no cases of instrumentation failure or pseudoarthrosis, demonstrating the mechanical stability of the expandable interbody device and percutaneous fusion construct. But perhaps most importantly, the long-term clinical outcomes in these patients demonstrate significant reductions in ODI scores, reflecting durable, meaningful, functional status improvements following this procedure.

\section{Complications and Technical Refinements}

The awake TLIF procedure evolved over the course of the first 100 cases. Growing familiarity with and increased efficiency in the procedure on the part of the surgeon and operative team have enabled multilevel cases to be performed within a safe operative time, as determined by our dedicated anesthesia team.

Changes to the medical and anesthetic protocol have been largely driven by experiences within this series, as described above. These have principally involved prophylaxis against epistaxis and emesis, both of which are of particular concern in a prone patient without a protected airway.

Operative complications, such as cage migration and endplate fracture, occurred predominantly in the first half of the cases of this series. We believe this distribution demonstrates an acceptable rate of technical mastery with the expandable interbody system we employ. Postoperative clinical assessment has also taught us the importance of timing when injecting local anesthetic agents along screw trajectories. We now consistently make an injection prior to incision in order to facilitate local diffusion of the liposomal bupivacaine solution by maintaining higher internal tissue pressure.

\section{Awake TLIF and Enhanced Recovery}

The surgical principles of ERAS - minimal soft-tissue destruction, reduced blood loss, and less reliance on intraoperative narcotic analgesia-are well reflected in our awake TLIF procedure. In fact, the development and increased utilization of this procedure at our institution predates our own ERAS program for lumbar fusion, which is now in its early implementation phase. It should be stressed, therefore, that the benefits we ascribe to awake TLIF in this study are solely attributable to the innovative procedure itself, as all cases in this series predate our ERAS protocol.

We believe that this supports the generalizability of our findings for surgeons considering the adoption of this technique at their own institutions. For any neurosurgeon seeking to achieve these operative and clinical results, especially in the setting of an ERAS program for spinal fusion, this procedure can easily serve as an MIS cornerstone within the additional pre- and postoperative ERAS interventions. We therefore anticipate further benefits in future patients who undergo this procedure in combination with nonsurgical ERAS components.

\section{Study Limitations}

This study has significant limitations. First, the sample size of 100 patients may not be adequate to fully assess the clinical efficacy of this procedure. However, compared with our previous reports we have a much greater number of patients included in this analysis, with long-term data for more than $80 \%$ of patients at the 1-year follow-up. Additionally, as a single-center study of patients treated by a single surgeon, the results must be validated across diverse practice environments and geographies. Second, many anesthesiologists may be reluctant to position patients prone without a protected airway. Over the course of this patient series, however, we improved the efficiency of the operation, owing to familiarity and experience with the procedure by the entire surgical and anesthesia team. Third, awake TLIF can only be applied in select cases, as endoscopic decompression can be limiting and indirect decompression can only achieve so much central canal enlargement for patients with severe or bilateral central canal stenosis. Finally, this technique requires off-label applications of expandable mesh cages, rhBMP-2, and liposomal bupivacaine, which must be considered despite their common practice in the United States.

\section{Conclusions}

The long-term results of our extended series of the first 100 patients to undergo awake endoscopic MIS-TLIF demonstrate operative and clinical outcomes comparable to those from our previous reports. We believe that our procedure provides patients with a safe and efficacious option for lumbar fusion without the morbidity associated with an open surgery. Further refinements in surgical technique and technologies will allow for improved success.

\section{References}

1. Al-Khouja LT, Baron EM, Johnson JP, Kim TT, Drazin D: Cost-effectiveness analysis in minimally invasive spine surgery. Neurosurg Focus 36(6):E4, 2014

2. Chang HK, Kolcun JPG, Chang PY, Wang MY: Enhanced Recovery After Surgery ${ }^{\text {Th }}$ awake minimally-invasive transforaminal lumbar interbody fusion: 2-dimensional operative video. Oper Neurosurg (Hagerstown) [epub ahead of print], 2018

3. Chin KR, Coombs AV, Seale JA: Feasibility and patientreported outcomes after outpatient single-level instrumented posterior lumbar interbody fusion in a surgery center: 
preliminary results in 16 patients. Spine (Phila Pa 1976) 40:E36-E42, 2015

4. Deyo RA, Mirza SK, Martin BI, Kreuter W, Goodman DC, Jarvik JG: Trends, major medical complications, and charges associated with surgery for lumbar spinal stenosis in older adults. JAMA 303:1259-1265, 2010

5. Eckman WW, Hester L, McMillen M: Same-day discharge after minimally invasive transforaminal lumbar interbody fusion: a series of 808 cases. Clin Orthop Relat Res 472:1806-1812, 2014

6. Kehlet H: Multimodal approach to control postoperative pathophysiology and rehabilitation. Br J Anaesth 78:606617, 1997

7. Lu VM, Kerezoudis P, Gilder HE, McCutcheon BA, Phan K, Bydon M: Minimally invasive surgery versus open surgery spinal fusion for spondylolisthesis: a systematic review and meta-analysis. Spine (Phila Pa 1976) 42:E177-E185, 2017

8. Pearson AM, Lurie JD, Tosteson TD, Zhao W, Abdu WA, Weinstein JN: Who should undergo surgery for degenerative spondylolisthesis? Treatment effect predictors in SPORT. Spine (Phila Pa 1976) 38:1799-1811, 2013

9. Rajaee SS, Bae HW, Kanim LE, Delamarter RB: Spinal fusion in the United States: analysis of trends from 1998 to 2008. Spine (Phila Pa 1976) 37:67-76, 2012

10. Rihn JA, Hilibrand AS, Zhao W, Lurie JD, Vaccaro AR, Albert TJ, et al: Effectiveness of surgery for lumbar stenosis and degenerative spondylolisthesis in the octogenarian population: analysis of the Spine Patient Outcomes Research Trial (SPORT) data. J Bone Joint Surg Am 97:177-185, 2015

11. Wainwright TW, Wang MY, Immins T, Middleton RG: Enhanced recovery after surgery (ERAS) - concepts, components, and application to spine surgery. Semin Spine Surg 30:104-110, 2018

12. Wang MY, Chang HK, Grossman J: Reduced acute care costs with the ERAS ${ }^{\circledast}$ minimally invasive transforaminal lumbar interbody fusion compared with conventional minimally invasive transforaminal lumbar interbody fusion. Neurosurgery 83:827-834, 2018
13. Wang MY, Chang PY, Grossman J: Development of an Enhanced Recovery After Surgery (ERAS) approach for lumbar spinal fusion. J Neurosurg Spine 26:411-418, 2017

14. Wang MY, Grossman J: Endoscopic minimally invasive transforaminal interbody fusion without general anesthesia: initial clinical experience with 1-year follow-up. Neurosurg Focus 40(2):E13, 2016

\section{Disclosures}

Dr. Wang reports that he is a consultant for Depuy-Synthes Spine, K2M, Spineology, and Stryker; receives royalties from Children's Hospital of Los Angeles, Depuy-Synthes Spine, Springer Publishing, and Quality Medical Publishing; is on the Advisory Board of Vallum; holds direct stock ownership in Innovative Surgical Devices; and receives support for a non-study-related clinical or research effort overseen by the US Department of Defense.

\section{Author Contributions}

Conception and design: Wang. Acquisition of data: Kolcun, Brusko. Analysis and interpretation of data: Kolcun, Basil. Statistical analysis: Basil. Drafting the article: Kolcun, Brusko, Basil. Critically revising the article: Kolcun, Epstein, Wang. Reviewed submitted version of manuscript: all authors. Approved the final version of the manuscript: Wang.

\section{Supplemental Information \\ Videos \\ Video Abstract. https://vimeo.com/321482729.}

\section{Correspondence}

John Paul G. Kolcun: University of Miami Miller School of Medicine, Miami, FL.jpk73@med.miami.edu. 